\title{
Oridonin enhances antitumor effects of doxorubicin in human osteosarcoma cells
}

\author{
Liliya Kazantseva $^{1,2,3} \cdot$ José Becerra ${ }^{1,2,3,4}$ (D) $\cdot$ Leonor Santos-Ruiz ${ }^{1,2,3,4}$ (D)
}

Received: 8 May 2021 / Revised: 13 August 2021 / Accepted: 17 August 2021 / Published online: 24 August 2021

(C) The Author(s) 2021

\begin{abstract}
Background Doxorubicin is the chemotherapeutic drug of choice in osteosarcoma treatment, but its cumulative administration causes dilated cardiomyopathy. Combination therapy represents a potential strategy to reduce the therapeutic dosage of the chemotherapeutic agent and minimize its side effects. The aim of this study was to evaluate the potential of oridonin, a natural product from the medicinal herb Rabdosia rubescens, to act in combination with doxorubicin for osteosarcoma treatment. To date, there are no reports of the simultaneous administration of both drugs in osteosarcoma therapy.

Methods The combined administration of different doses of oridonin and doxorubicin, as compared with the drugs alone, were tested in an in vitro model of osteosarcoma. The synergistic effect of the drugs on cell death was assessed by alamar$B l u e^{\mathrm{TM}}$ and by CompuSyn software. Early and late apoptosis markers (JC-1 fluorescence and Annexin V immunofluorescence), as well as the production of reactive oxygen species, were evaluated by flow cytometry. Western blot was used to assess the expression of anti-apoptotic proteins.

Results Oridonin and doxorubicin presented a synergistic cytotoxic effect in osteosarcoma cells. In the presence of subcytotoxic concentrations of the natural product, there was an increased accumulation of intracellular doxorubicin, increased levels of reactive oxygen species (ROS), alteration of mitochondria membrane potential and a higher rate of apoptosis.

Conclusion The combined use of oridonin and doxorubicin could help to reduce the clinical dosage of doxorubicin and its dangerous side effects.
\end{abstract}

Keywords Osteosarcoma $\cdot$ Doxorubicin $\cdot$ Oridonin $\cdot$ Synergism $\cdot$ Combination therapy $\cdot$ Cardioprotection

\begin{tabular}{|c|c|}
\hline Abbre & viations \\
\hline DOX & Doxorubicin \\
\hline ORI & Oridonin \\
\hline ROS & Reactive Oxy \\
\hline
\end{tabular}

Leonor Santos-Ruiz

lsantos@uma.es

1 Andalusian Centre for Nanomedicine and Biotechnology-BIONAND, Universidad de Málaga, Parque Tecnológico de Andalucía, C/ Severo Ochoa, 35, 29590 Campanillas Málaga, Spain

2 Centro de Investigación Biomédica en Red, Biotecnología, Biomateriales y Nanomedicina (CIBER-BBN), Madrid, Spain

3 Instituto de Investigación Biomédica de Málaga-IBIMA, Málaga, Spain

4 Departamento de Biología Celular, Genética y Fisiología Facultad de Ciencias, Universidad de Málaga, Campus de Teatinos, 29071 Málaga, Spain

\section{Combination Index \\ $\mathrm{CD}_{50} \quad 50 \%$ Cell Death}

\section{Introduction}

Osteosarcoma is a bone tumor characterized by the presence of differentiated osteoblasts producing immature osteoid matrix. It is one of the most common cancers affecting children and adolescents, and has a second peak of incidence after the age of $50[1,2]$.

Doxorubicin (DOX) is possibly the most commonly used drug alone or in combination with high-dose methotrexate and cisplatin for osteosarcoma treatment $[2,3]$. It is an anthracycline that achieves its therapeutic activity through DNA intercalation, leading to inhibition of topoisomerase-II function. Its efficiency, however, is limited by presence of a life-threatening side effect in the form of cardiotoxicity, which depends on cumulative dosing and can drive to congestive heart failure later in life [2, 4-6]. 
There is a growing interest in combination therapy that is based on administration of two or more drugs acting on different target sites, making it difficult for cancer cells to mutate and adapt to novel conditions. The synergistic effects observed between different drug types may drive to a reduction of drug dosing, toxicity, resistance, and to an increase of therapeutic efficiency [2, 7-9].

Oridonin (ORI) is a diterpenoid isolated from Rabdosia rubescens, (Hemsl.), H.Hara, a medicinal herb that has been used to treat esophageal cancer by native people of Henan province, in China [10,11]. This natural product is getting much attention for having anti-angiogenic properties and ability to inhibit growth and metastasis in different types of cancer, such as liver, colorectal and breast [12-15]. There are very few studies about the possibilities of ORI in osteosarcoma treatment, but these have shown promising results [16-18]. Synergy was found in combination with DOX against aggressive breast cancer, suggesting that the combined use of both drugs could help to decrease therapeutic DOX doses, thus reducing as well its secondary effects, like cardiotoxicity [15].

In the present study, we aimed to gain insight on the potential of a DOX plus ORI combination therapy for osteosarcoma treatment, by focusing on an in vitro model of pediatric osteosarcoma. We found that DOX and ORI possess a synergistic effect, as co-administration of both drugs significantly increased osteosarcoma cell death as compared with the drugs administered alone. Our data point to the possibility of using ORI as an adjunct in DOXbased chemotherapy treatments for osteosarcoma.

\section{Materials and methods}

\section{Reagents}

Unless otherwise stated, all reagents were purchased from Sigma-Aldrich (St. Louis, Missouri, USA).

\section{Cell culture}

Saos-2 cell line, an osteogenic sarcoma derived from a primary osteosarcoma of an 11-year-old girl, was purchased from the European Collection of Authenticated Cell Cultures (ECACC 89,050,205) [19]. It was maintained in McCoy's 5A medium, supplemented with $15 \%$ heat-inactivated Fetal Bovine Serum (FBS), and $2 \mathrm{mM} \mathrm{L}$-glutamine. It was incubated at $37^{\circ} \mathrm{C}$ in a humidified atmosphere with $5 \% \mathrm{CO}_{2}$ (standard culture conditions).

\section{Cell viability}

Cell viability was assessed using alamarBlue ${ }^{\mathrm{TM}}$ assay from Invitrogen (Carlsbad, California, USA). Cells were seeded at a density of 20,000 cells/well in $100 \mu \mathrm{L}$ of the corresponding medium, in a 96-well plate. To let cell attachment, the plate was incubated in standard culture conditions for $24 \mathrm{~h}$. After this time, Saos- 2 was treated with different concentrations of DOX $(0.1-10 \mu \mathrm{M})$, or ORI $(10-40 \mu \mathrm{M})$, administered in the culture medium. After $48 \mathrm{~h}$ of incubation, the treatment medium was removed and the cells were rinsed with Phosphate Buffered Saline (PBS). Cell viability was measured by incubating the cells for $4 \mathrm{~h}$ in $20 \%$ alamarBlue $^{\mathrm{TM}}$ dissolved in culture medium. Then, the alamarBlue ${ }^{\mathrm{TM}}$ fluorescence emission was measured on a LS55 fluorometer plate reader (Perkin Elmer, Waltham, Massachusetts, USA) at $530 \mathrm{~nm}$-excitation and $590 \mathrm{~nm}$-emission wavelengths. The obtained data allowed selecting the concentration that achieved $50 \%$ of cell death $\left(\mathrm{CD}_{50}\right)$ for each drug. Then, combined drug therapy was evaluated by treating cells with different combinations of DOX $\left(\mathrm{CD}_{50}, \mathrm{CD}_{50} / 2\right.$ and $\left.\mathrm{CD}_{50} / 10\right)$ and ORI $\left(\mathrm{CD}_{50}, \mathrm{CD}_{50} / 2\right.$ and $\left.\mathrm{CD}_{50} / 10\right)$ and assessing viability as described. Six wells were used for each condition in all experiments. Experiments were repeated thrice.

The interaction between DOX and ORI was quantified through the Chou-Talalay method [20]. For this, the combination index (CI) was calculated using the CompuSyn software (ComboSyn, Inc., Paramus, New Jersey, USA), where $\mathrm{CI}<1$ indicated synergism, $\mathrm{CI}=1$ additive effect and $\mathrm{CI}>1$ antagonism.

\section{Cell uptake of DOX}

To establish if there are changes in the intracellular accumulation of DOX due to the presence of ORI, intracellular DOX fluorescence was measured by flow cytometry. For this purpose, Saos-2 cells were seeded at a density of 150,000 cells/well in 12-well plates and incubated for $24 \mathrm{~h}$ in standard culture conditions to allow cell attachment. Then, Saos- 2 cells were treated for $2 \mathrm{~h}$ with $2.5 \mu \mathrm{M}$ DOX, $10 \mu \mathrm{M}$ ORI and $2.5 \mu \mathrm{M}$ DOX $+10 \mu \mathrm{M}$ ORI. These concentrations were chosen based on their calculated CIs. Untreated cells were used as control. The treatment conditions were performed in triplicate. After incubation, cells were rinsed with PBS, collected by trypsinization and centrifuged at $1800 \mathrm{rpm}$ for $5 \mathrm{~min}$. The supernatant was removed and the pellet resuspended in $1 \mathrm{~mL}$ of cold PBS with $2 \%$ of heat-inactivated FBS. Then, the cells were transferred to $5 \mathrm{~mL}$ tubes and analyzed with a Gallios flow cytometer (Beckman Coulter, Brea, California, USA). 
DOX fluorescence was detected in FL3 channel. For each sample 25,000 events were collected. Experiments were repeated thrice.

\section{Cytotoxicity-related cellular events}

To understand how the drugs under study induced cytotoxicity, mitochondrial membrane potential, apoptosis, and reactive oxygen species were assayed. For this purpose, Saos-2 cells were seeded into 12-well multi-well plates, at a density of 150,000 cells/well, in normal medium, and incubated for $32 \mathrm{~h}$ in standard culture conditions. Then, they were treated for $16 \mathrm{~h}$ with $2.5 \mu \mathrm{M}$ DOX, $10 \mu \mathrm{M}$ ORI and combined DOX + ORI $(2.5 \mu \mathrm{M}$ and $10 \mu \mathrm{M}$, respectively). After drug exposure, the treatment medium was removed and cells were washed with PBS, collected by trypsinization and pelleted by centrifugation at $1800 \mathrm{rpm}$ for $5 \mathrm{~min}$. The pellet was then treated as described below for each assay. Cells without any treatment were used as controls. Each condition was done in triplicate. Experiments were replicated thrice.

\section{Mitochondrial membrane potential}

Changes of the mitochondrial inner membrane electrochemical potential were detected with Mitochondria Staining Kit, according to the manufacturer's instructions. Briefly, pelleted cells were resuspended in $1 \mathrm{~mL}$ of JC-1 staining mixture, $(5 \mu \mathrm{g} / \mathrm{mL}$ JC-1 dissolved in staining buffer, according to the manufacturer's instructions), and incubated for $20 \mathrm{~min}$, in standard culture conditions. Cells treated with valinomycin were used as a control for mitochondrial gradient dissipation. For this purpose, valinomycin $(0.2 \mu \mathrm{g} / \mathrm{mL}$ final concentration) was added to the JC-1 staining mixture. After incubation in JC-1 staining solution, the cells were pelleted by centrifugation, the supernatant removed, and the pellet resuspended in ice-cooled JC-1 staining buffer. The fluorescence of the stained cells was measured by flow cytometry. JC-1 monomers were detected in the FL1 channel and JC-1 aggregates were detected in the FL2 channel. For each sample 10,000 events were collected.

\section{Apoptosis assay}

Apoptosis was detected by flow cytometry using Annexin V-FITC/PI Apoptosis Detection kit (Elabscience, Houston, Texas, USA). Manufacturer's instructions were followed with some modifications. Presence of DOX inside the cells was overlapping with PI in FL3 channel, leading to the overestimation of the dead cells. For this reason, Hoechst 33258 was used instead. Pelleted cells were resuspended, and incubated for $20 \mathrm{~min}$, in $100 \mu \mathrm{L}$ Annexin $\mathrm{V}$ binding buffer containing $2.5 \mu \mathrm{L}$ Annexin V-FITC conjugate and $2.5 \mu \mathrm{L}$ Hoechst staining solution. Then, $400 \mu \mathrm{L}$ binding buffer was added and cells were transferred to FC tubes to be analyzed by flow cytometry. For each sample 10,000 events were collected. The fluorescence of Annexin V-FITC was detected in FL1 channel, and Hoechst 33258 in FL9. Cells in the lower left quadrant (Annexin V-FITC - /Hoechst 33258-) were considered to be live cells. Early apoptotic event (Annexin V-FITC + I Hoechst $33258-$ ) was considered when cells were located in the lower right quadrant. Late apoptotic event (Annexin V-FITC +/Hoechst 33258+) was considered if cells localized in the upper right quadrant. Finally, necrosis (Annexin V-FITC - /Hoechst $33258+$ ) was considered when cells were localized in the upper left quadrant. The apoptotic index was calculated by combining the data of early and late apoptosis. Data were normalized to the control, which percentage of apoptosis was considered as $100 \%$.

\section{Reactive oxygen species (ROS) assay}

The intracellular ROS level was measured by flow cytometry using the Fluorometric Intracellular ROS kit. Manufacturer instructions were followed with some modifications. Pelleted Saos- 2 cells were resuspended in $500 \mu \mathrm{L}$ of assay buffer and $1 \mu \mathrm{L} 500 \mathrm{X}$ ROS detection reagent stock solution was added to each tube. The cells were incubated for $1 \mathrm{~h}$ at $37{ }^{\circ} \mathrm{C}$ in a humidified atmosphere with $5 \% \mathrm{CO}_{2}$. Fluorescence intensity was measured in the FL6 channel of a flow cytometer. For each sample, 10,000 events were collected.

\section{Western Blot analysis}

Cells were harvested with a scraper, and lysed in RIPA lysis buffer containing a mix of protease inhibitors. Bradford protein assay was used to determine the total protein concentration. Proteins were separated on SDS-PAGE and transferred onto nitrocellulose membrane. Non-specific antibody binding was blocked with 5\% non-fat milk. The membrane was incubated with the following primary antibodies: anti-Mcl-1 (1:1000), anti-Bcl-2 (1:1000), anti-BclXL $(1: 1000)$ and anti- $\beta$-actin $(1: 2000)$ overnight at $4{ }^{\circ} \mathrm{C}$. Subsequently, a horseradish peroxidase-conjugated secondary antibody (1:5000) was incubated at room temperature for $2 \mathrm{~h}$. Finally, the protein bands were visualized with ECL Western Blotting Substrate and photographed with ChemiDoc XRS + (Bio-Rad). Experiments were performed thrice. Semi-quantification of band densitometry was carried out by measuring band density with ImageJ software. All bands in a gel were normalized against the gel background, and then Mcl-1, Bcl-2 and Bcl-XL bands were normalized against their corresponding $\beta$-actin loading controls. 


\section{Statistical analysis}

Quantitative data were graphed and statistically analyzed with Excel 16.0 (ed. 2016, Microsoft, Redmond, Washington, USA), and Prism 6.0c (ed. 2013, GraphPad Software Inc., San Diego, California, USA). Shapiro-Wilk test was used for normality evaluation of the data. The results were analyzed by a two-way analysis of variance (ANOVA) followed by Tukey's multiple comparison test. Values with $p<0.05$ were considered statistically significant. Data are represented as mean $\pm \mathrm{SD}$. Western-blot semi-quantitative data were analyzed by Kruskal-Wallis test.

\section{Results and discussion}

In the present study, we aimed to evaluate the possibility of enhancing the effect of DOX, a common drug in the treatment of osteosarcoma, by combining it with ORI. This combination has not been studied yet for this type of cancer. Saos-2 cells were treated with 7 different concentrations of DOX and ORI in order to select the concentration that achieved a $50 \%$ of cell death $\left(\mathrm{CD}_{50}\right)$ for each drug after $48 \mathrm{~h}$ of exposure. Our results confirmed that both drugs, individually administered, reduced cell viability in a dosedependent manner (Fig. 1A and B). DOX showed a more toxic effect than ORI, with the drug concentration causing $50 \%$ cell death being determined as CD50 $\approx 5 \mu \mathrm{M}$ for DOX, and $\mathrm{CD} 50 \approx 20 \mu \mathrm{M}$ for ORI.

Based on the cytotoxicity induced by the drugs alone, the effects of different DOX doses $\left(\mathrm{CD}_{50}, \mathrm{CD}_{50} / 2\right.$ and $\left.\mathrm{CD}_{50} / 10\right)$ $\left(\mathrm{CD}_{50}, \mathrm{CD}_{50} / 2\right.$ and $\left.\mathrm{CD}_{50} / 10\right)$ combined with different doses of ORI $\left(\mathrm{CD}_{50}, \mathrm{CD}_{50} / 2\right.$ and $\left.\mathrm{CD}_{50} / 10\right)$ were evaluated. In general, simultaneous treatment produced a higher cytotoxic effect compared to drugs alone (Fig. 2A). The synergistic effect of DOX and ORI in Saos-2 cytotoxicity was confirmed by calculating the Combination Index (CI; Fig. 3A), which is a quantitative definition of synergism, additive and antagonism [20]. These results thus confirm that combination with ORI would allow an increased chemotherapeutic effect of DOX, and might then represent a step forward for the reduction of DOX unwanted side effects.

We decided to carry on our experiments with $2.5 \mu \mathrm{M}$ DOX $+10 \mu \mathrm{M}$ ORI. This dosing corresponds to a twofold reduction over DOX $\mathrm{CD}_{50}$ (Fig. 2B), combined with a minimally cytotoxic dose of ORI (Figs. 2B shows that cells treated with $10 \mu \mathrm{M}$ ORI are almost $100 \%$ viable). After $16 \mathrm{~h}$ of treatment, the selected DOX + ORI pair presented enhanced mortality, with only few cells still adhered to the plate, as compared to the rest of the controls (Fig. 3B). We could confirm that apoptosis, as checked by Annexin V-FITC/Hoechst 33258 staining, was induced in cultures by DOX and ORI alone, where the apoptotic index was significantly higher in cells treated with the combined drugs (Fig. 4A and B).

From previous studies in osteosarcoma, it is known that ORI induces apoptosis through mitochondrial toxicity [17]. Accordingly, after $16 \mathrm{~h}$ of treatment, significant changes in mitochondrial potential were observed in Saos-2 cells treated with DOX + ORI, where a marked switch from red to green fluorescence indicated a mitochondrial membrane depolarization, which is an early feature of cell death (Fig. 4C and D) [21].

To understand how ORI enhanced the toxicity of the chemotherapeutic drug, DOX cell uptake was studied using flow cytometer, on account of the red fluorescence of DOX. After $2 \mathrm{~h}$ of exposure to DOX plus ORI, Saos-2 cells presented a greater shift in DOX fluorescence as compared to DOX alone, indicating that ORI had increased the intracellular uptake of DOX (Fig. 5A and B). Hence, it is reasonable to hypothesize that intracellular DOX accumulation induced by ORI might have caused mitochondrial
A

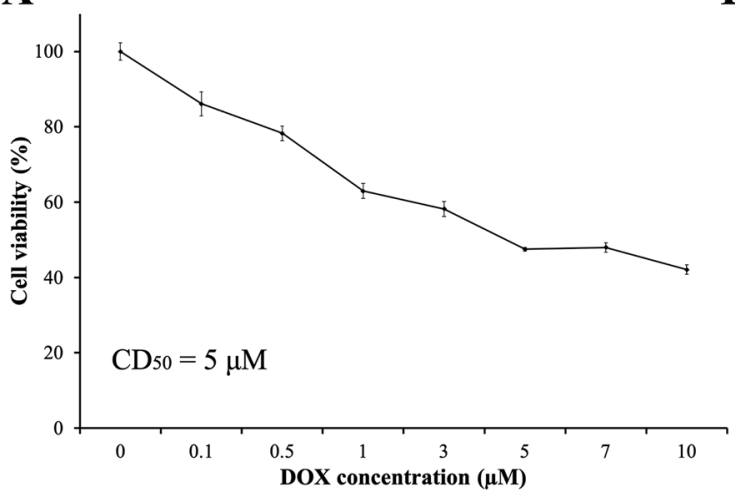

B

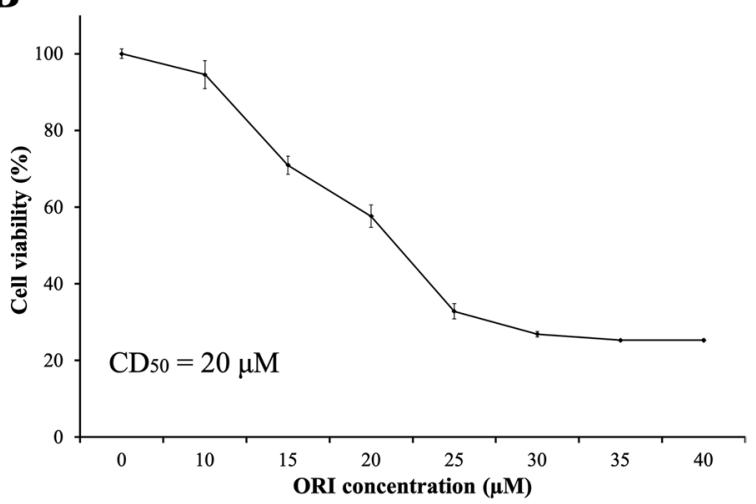

Fig. 1 Toxicity evaluation of DOX and ORI. Saos-2 viability was assessed after $48 \mathrm{~h}$ of exposure to different concentrations of DOX (A) and ORI (B) using alamarBlue ${ }^{\mathrm{TM}}$ assay. Data are represented as mean $\pm \mathrm{SD}$ 
$\mathbf{A}$

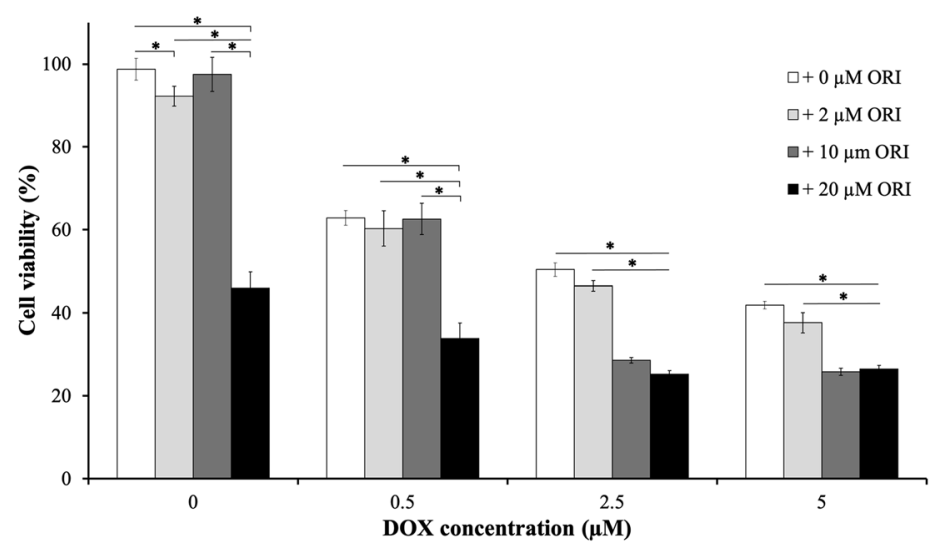

B

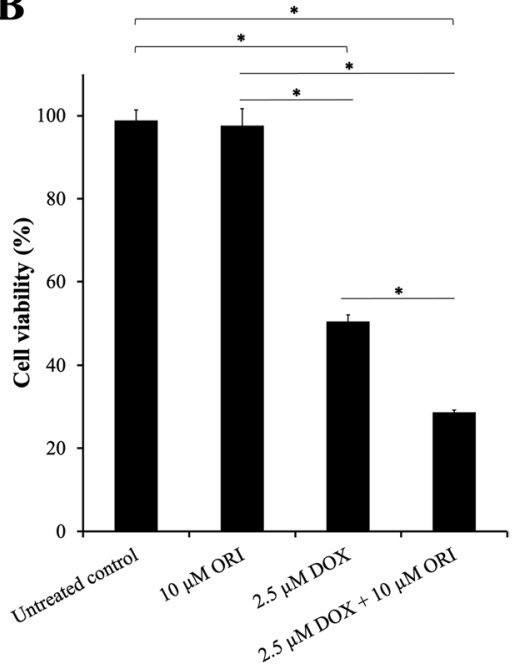

Fig. 2 Toxicity evaluation of DOX and ORI combination. Saos-2 cells were exposed for $48 \mathrm{~h}$ to concentrations of DOX and ORI corresponding to their $\mathrm{CD}_{50}, \mathrm{CD}_{50} / 2$ and $\mathrm{CD}_{50} / 10$; as well as to combinations of both drugs (A). Separate representation of the selected concentration of DOX, ORI and combination of both chosen for the posterior experiments (B). Cell viability was assessed by alamar$B l u e^{\mathrm{TM}}$ assay. Analysis was done using two-way ANOVA test followed by Tukey's multiple comparisons test. Asterisk denotes statistical significance. Significant effect was observed with two-way ANOVA: $F_{3,80}=680.9, p<0.0001$ for main effect and $F_{9,80}=100.6, p<0.0001$ for interaction. In $0 \mu \mathrm{M}$ DOX group, the post hoc analysis indicated significance in the following subgroups: $p=0.0004$ between $0 \mu \mathrm{M}$ ORI and $2 \mu \mathrm{M} \mu \mathrm{M}$ ORI; $p=0.0074$ between $2 \mu \mathrm{M}$ ORI and $10 \mu \mathrm{M}$ ORI; $p<0.0001$ in the rest of the subgroups. In $0.5 \mu \mathrm{M}$ DOX, the post hoc analysis indicated significance in the following subgroups: $p<0.0001$ in subgroups with the asterisk. In $2.5 \mu \mathrm{M}$ DOX, the post hoc analysis indicated significance in the following subgroups: $p=0.0464$ between $0 \mu \mathrm{M}$ ORI and $2 \mu \mathrm{M} \mu \mathrm{M}$ ORI; $p<0.0001$ in the rest of the subgroups. In $5 \mu \mathrm{M}$ DOX, the post hoc analysis indicated significance in the following subgroups: $p=0.0255$ between $0 \mu \mathrm{M}$ ORI and $2 \mu \mathrm{M} \mu \mathrm{M}$ ORI; $p<0.0001$ in the rest of the subgroups. Data are represented as mean $\pm \mathrm{SD}$
A

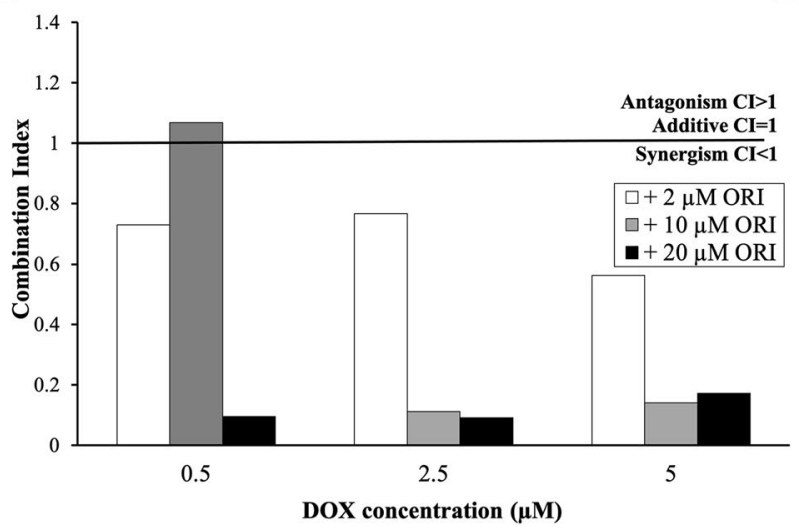

B

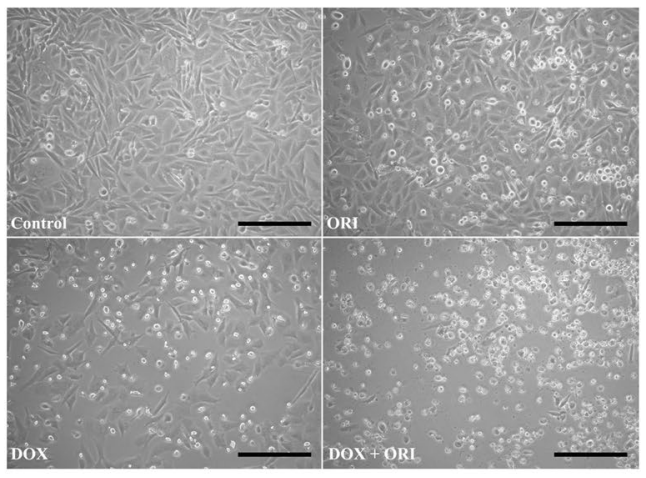

Fig. 3 Effects of ORI, DOX and the combination of both on Saos-2. Combination Index (A). Morphology of Saos-2 cultures after $16 \mathrm{~h}$ of treatment with $2.5 \mu \mathrm{M}$ DOX, $10 \mu \mathrm{M}$ ORI, and the combination of

toxicity and this, in turn, produced cellular apoptosis. In general, our results are in accordance with the findings of $\mathrm{Li}$ et al. where synergy of ORI was found in combination with DOX against aggressive breast cancer, suggesting that the combined used of both drugs could help to both was observed using a 10X objective in an inverted microscope (B). Scale bar $=50 \mu \mathrm{m}$ decrease therapeutic DOX doses, thus reducing as well its secondary effects, such as cardiotoxicity [15].

We further investigated the possible mediator that could be implicated in the triggered apoptosis. It is known DOX induces apoptosis through ROS induction, even in cells 
$\mathbf{A}$

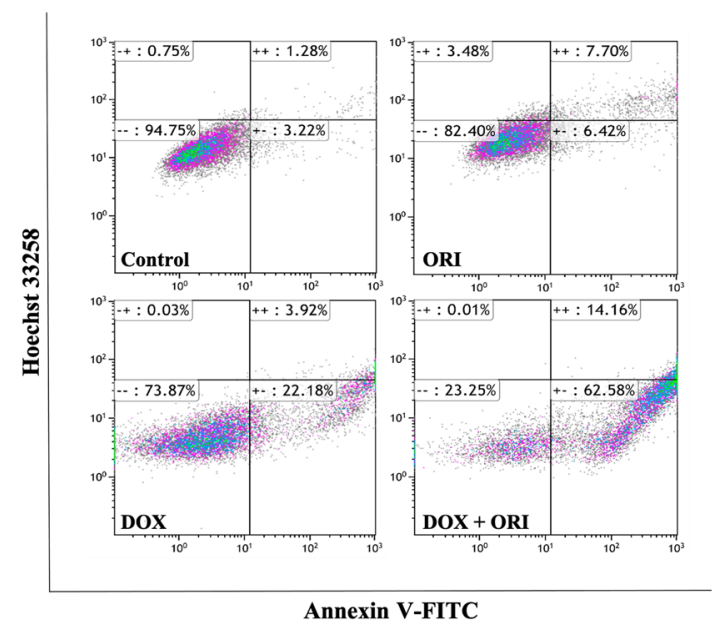

C

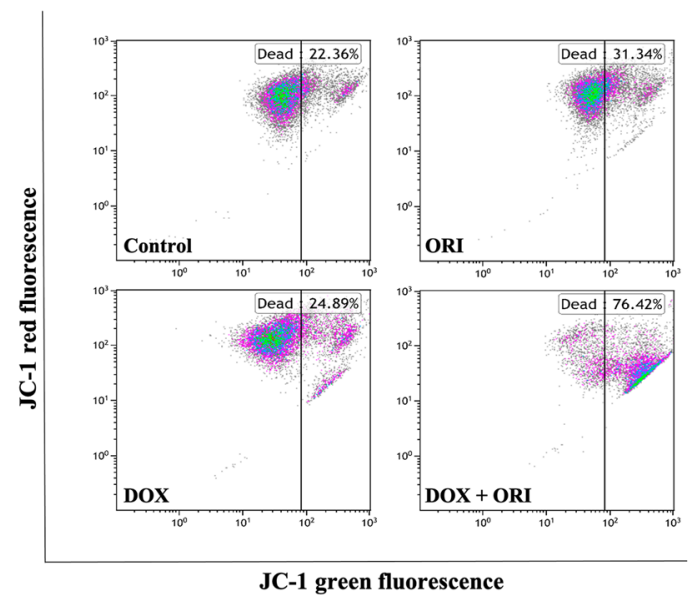

Fig. 4 Effects of ORI, DOX and the combination of both on Saos-2 cell death. Annexin V-FITC and Hoechst 33258 fluorescent signals, as measured by flow cytometry (A). Apoptotic index as determined by Annexin V-FITC/Hoechst 33258 staining (B). In each group, the data of early and late apoptosis were combined and normalized to the control, which percentage of apoptosis was considered as $100 \%$. Significance was observed with two-way ANOVA $\left(F_{3,6}=217.2\right.$; $p<0.0001$ ). Post hoc analysis indicated significant effect in the following groups: $p<0.0001$ in DOX+ORI vs. Control/ORI/DOX; $p=0.0432$ in Control vs. ORI; $p=0.0021$ in Control vs. DOX. JC-1 red and green fluorescent signals in each experimental group, as

like Saos-2 that lack functional p53 [22]. Additionally, cancer cells are characterized by elevated levels of oxidative stress, meaning that chemotherapeutic agents that can promote a further increase in ROS production are a preferential strategy against cancer [23]. To determine its participation in mitochondrial-mediated cell death, we studied intracellular ROS levels in Saos-2 osteosarcoma cells. Both DOX and ORI were able to increase intracellular ROS levels, as compared to untreated controls (Fig. 5C and D). These results point to ROS induction being responsible for the activation of the cascade leading
B

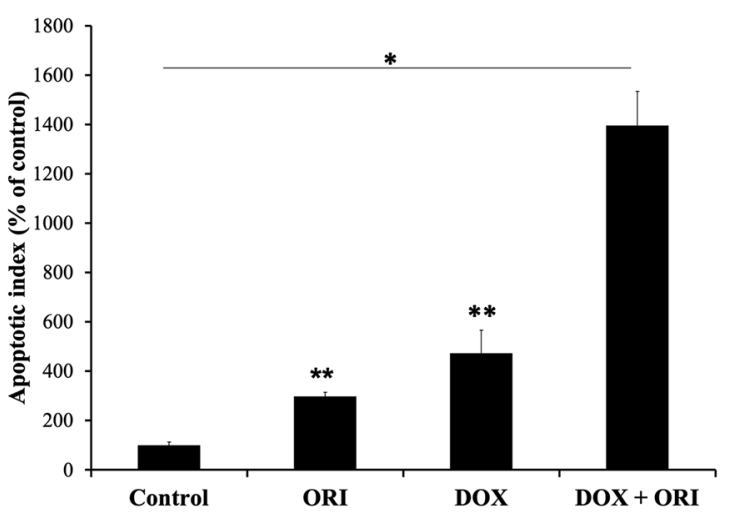

D

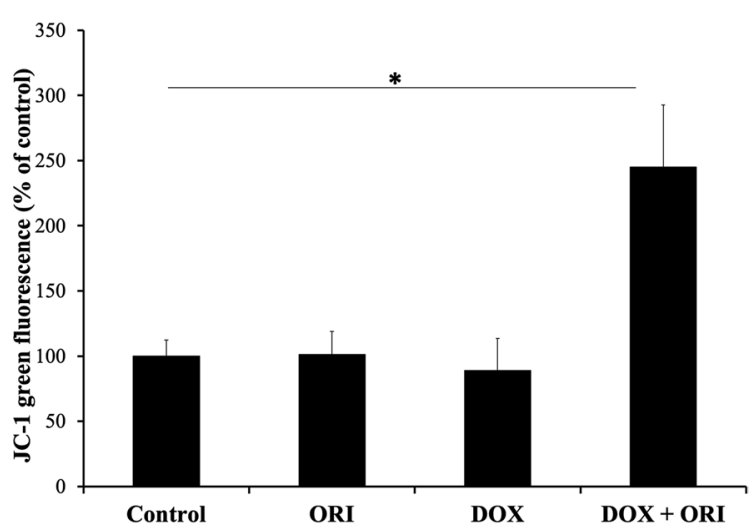

measured by flow cytometry $(\mathbf{C})$. Mitochondrial membrane potential as determined by quantification of JC-1 fluorescence. Significance was observed with two-way ANOVA $\left(F_{3,6}=30.01 ; p=0.0005\right)$. Post hoc analysis indicated significant effect in the following groups: $p=0.0011$ in DOX + ORI $v s$. Control; $p=0.0012$ in DOX + ORI $v s$. ORI; $p=0.0008$ in DOX + ORI vs. DOX (D). One asterisk shows statistical significance between DOX + ORI and the rest of the treatment groups, while two asterisks is compared to the control (ORI/DOX vs. Control) according to a two-way ANOVA test followed by Tukey's multiple comparison test. Data are represented as mean $\pm \mathrm{SD}$

to cell death in Saos- 2 cells, as has been observed in other studies [22, 24].

Then, we studied the effects of each treatment on the expression of Mcl-1, Bcl-2 and Bcl-XL, anti-apoptotic members of the Bcl-2 protein family, by Western Blot analysis (Fig. 6). Mcl-1 was only detected in cells exposed to ORI alone. Bcl-2 and Bcl-XL were observed in both control and experimental conditions, being their level lower in DOX + ORI-treated cells than in cells treated with DOX alone. This can account for the higher level of apoptosis observed in DOX + ORI-treated cells, as compared 
A

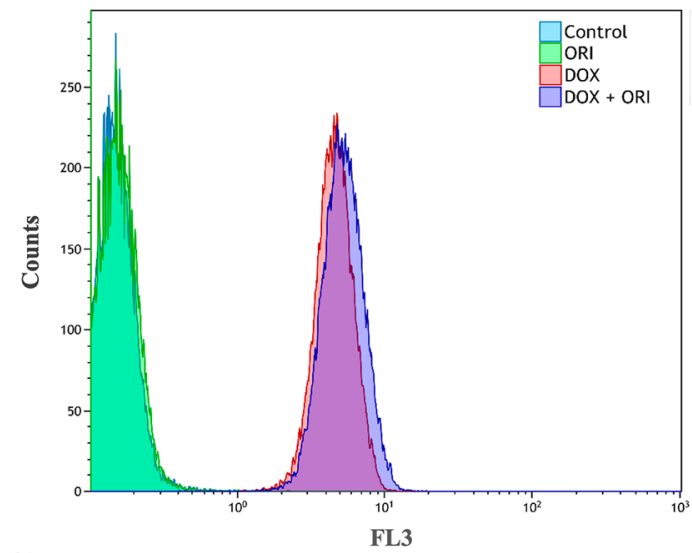

C

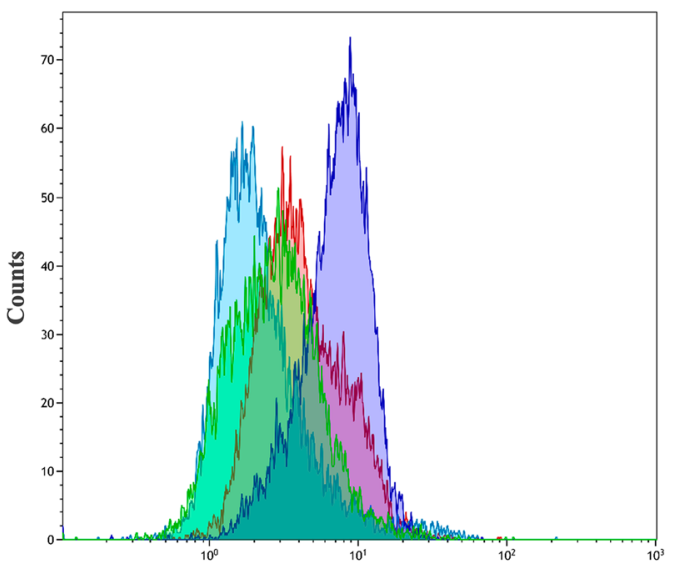

B

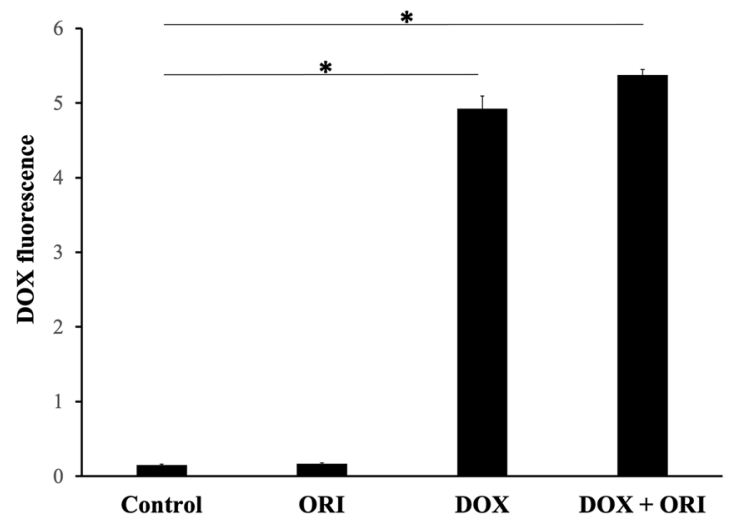

D

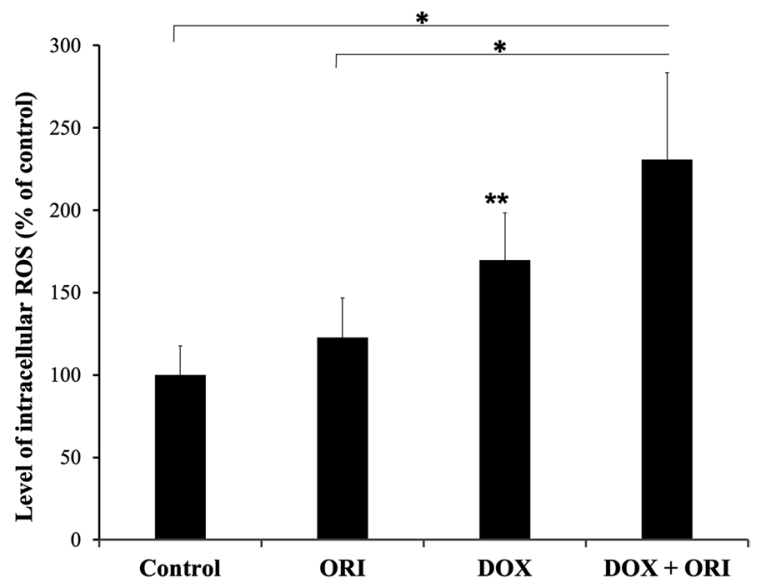

Fig. 5 Effects of ORI, DOX and the combination of both on Saos-2. DOX fluorescent signal in each experimental group, as measured by flow cytometry (A). Quantification of DOX red fluorescence signal in control and drugs-treated Saos- 2 cells after $2 \mathrm{~h}$ of treatment. One asterisk shows statistical significance between DOX/DOX + ORI and the rest of the treatment groups. Significant effect was observed with two-way ANOVA $\left(F_{3,6}=2589 ; p<0.0001\right)$. Post hoc analysis indicated significance in the following groups: $p<0.0001$ in DOX + ORI vs. Control/ORI and $p=0.0057$ in DOX + ORI vs. DOX; $p<0.0001$ in DOX vs. Control/ORI (B). ROS fluorescent signal in each experi- mental group, as measured by flow cytometry (C). Quantification of intracellular ROS levels (D). One asterisk shows statistical significance between DOX + ORI and Control/ORI, while two asterisks is DOX compared to the Control. Significant effect was observed with two-way ANOVA $\left(F_{3,6}=18.13 ; p=0.0021\right)$. Post hoc analysis indicated significance in the following groups: $p=0.0020$ in DOX +ORI vs. Control; $p=0.0054$ in DOX + ORI vs. ORI; $p=0.0414$ in DOX vs. Control (D). Analysis was done using two-way ANOVA test followed by Tukey's multiple comparison test. Data are represented as mean $\pm \mathrm{SD}$ with those that received the chemotherapeutic drug alone. In contrast, ORI exposed cells presented reduced induction of ROS and less cell death, suggesting a link between oxidative stress and apoptosis in our model. These results agree with the higher levels of Mcl-1, Bcl-2 and Bcl-XL observed in ORI-treated cells. Demelash et al. found that Mcl-1 expression prevented ROS formation, through inhibition of NOX4, a NADPH oxidase, and its posterior translocation to the mitochondria [25]. In our model, Mcl-1 expression could be promoting the survival of Saos-2 cells treated with low doses of ORI. Possibly, higher doses would inhibit the activation of Mcl-1 leading to apoptosis, as observed from Fig. 6. However, further studies will be needed to confirm and elucidate the mechanism of ROS implication in all the experimental groups.

In this study, our results confirmed that combination with ORI would allow both an increased chemotherapeutic effect of DOX, and a drastic reduction of its therapeutic dose. In a breast cancer model the simultaneous administration of both drugs induced apoptosis by reducing the expression of Bcl-2 and Survivin, while increasing the expression of Bax. Moreover, DOX and ORI combination was shown to suppress angiogenesis possibly through the inhibition of VEGFR2-mediated signaling pathway [15]. Several studies demonstrated ORI to enhance the cytotoxicity of different chemotherapeutic drugs that could be 

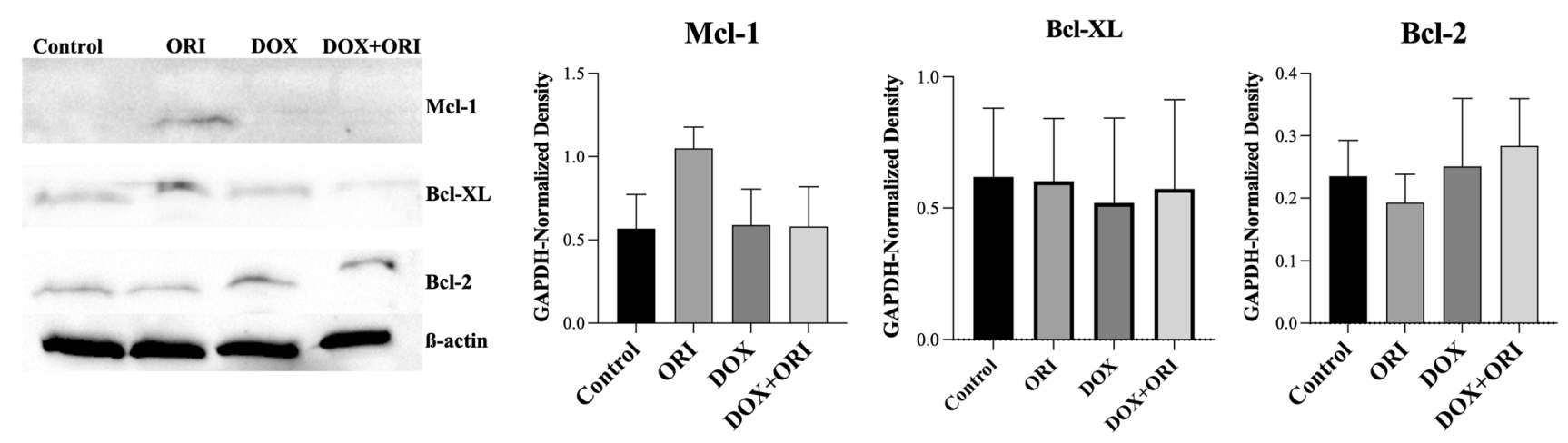

Fig. 6 Effects of ORI, DOX and the combination of both on the expression of apoptosis-related proteins in Saos-2. The expression of Mcl-1, Bcl-2 and Bcl-XL was detected after Saos-2 were exposed to $\mathrm{DOX}+\mathrm{ORI}$ for $16 \mathrm{~h}$. $\beta$-actin was used as internal reference. The gel

explained by more than one mechanism and the cellular pathways affected could be specific or shared between different types of tumors. In hepatocellular carcinoma, a synergistic effect was observed between cisplatin and ORI, which was produced by inhibition NF- $\mathrm{KB}$ transcription, one of the major regulators of innate and adaptive immunity [26]. In gastric cancer ORI was reported to reverse cisplatin resistance by suppressing P-gp expression, which is involved in drug efflux [27].

In summary, our results provide a first insight of DOX plus ORI combination therapy for osteosarcoma treatment. The simultaneous administration of both drugs induced apoptosis through mitochondrial toxicity, possibly mediated by ROS. By increasing DOX cytotoxic effect in osteosarcoma cells, ORI could allow reducing DOX doses in osteosarcoma treatment.

Acknowledgements Authors would like to thank Luisa Macías Ramírez for her kind technical assistance with flow cytometry.

Author contributions Conceptualization, LS-R; methodology, LK; software, LK and LS-R; formal analysis, LK and LS-R; investigation, LK and LS-R; resources, LS-R and JB; writing-original draft preparation, LK; writing - review and editing, LK and LS-R; visualization LK and LS-R; supervision, LS-R; project administration, JB and LS-R; funding acquisition, JB and LS-R All authors have read and agreed to the published version of the manuscript.

Funding This project received funding from the European Union's Horizon 2020 research and innovation program under the Marie Skłodowska-Curie grant agreement $\mathrm{N}^{\circ}$ 713721; Ministerio de Economía y Competitividad (MINECO; grant number BIO201566266-R); Instituto de Salud Carlos III (grant numbers CB06/06/1015 and RD16/0011/0022); Consejería de Salud y Asuntos Sociales de la Junta de Andalucía (grant number PI-0032-2016).

\section{Declarations}

Conflict of interest The authors declare no conflict of interest. images depict the more representative blots out of three experiments. The graphs represent protein densitometry measured in the three gels as mean \pm SD. Data were analyzed by a Kruskal-Wallis and Dunn's multiple comparisons test

Open Access This article is licensed under a Creative Commons Attribution 4.0 International License, which permits use, sharing, adaptation, distribution and reproduction in any medium or format, as long as you give appropriate credit to the original author(s) and the source, provide a link to the Creative Commons licence, and indicate if changes were made. The images or other third party material in this article are included in the article's Creative Commons licence, unless indicated otherwise in a credit line to the material. If material is not included in the article's Creative Commons licence and your intended use is not permitted by statutory regulation or exceeds the permitted use, you will need to obtain permission directly from the copyright holder. To view a copy of this licence, visit http://creativecommons.org/licenses/by/4.0/.

\section{References}

1. Kansara M, Teng MW, Smyth MJ, Thomas DM. Translational biology of osteosarcoma. Nat Rev Cancer. 2014;14(11):722-35.

2. González-Fernández Y, Imbuluzqueta E, Zalacain M, Mollinedo F, Patiño-García A, Blanco-Prieto MJ. Doxorubicin and edelfosine lipid nanoparticles are effective acting synergistically against drug-resistant osteosarcoma cancer cells. Cancer Lett. 2017;388:262-8.

3. Anderson ME. Update on Survival in osteosarcoma. Orthop Clin North Am. 2016;47(1):283-92.

4. Janeway KA, Grier HE. Sequelae of osteosarcoma medical therapy: a review of rare acute toxicities and late effects. Lancet Oncol. 2010;11(7):670-8.

5. Thorn CF, Oshiro C, Marsh S, Hernandez-Boussard T, McLeod $\mathrm{H}$, Klein TE, et al. Doxorubicin pathways: pharmacodynamics and adverse effects. Pharmacogenet Genom. 2011;21(7):440-6.

6. Yang Y, Niu X, Zhang Q, Hao L, Ding Y. The efficacy of abraxane on osteosarcoma xenografts in nude mice and expression of secreted protein, acidic and rich in cystein. Am J Med Sci. 2012;344(3):199-205.

7. Jia J, Zhu F, Ma X, Cao Z, Cao ZW, Li Y, et al. Mechanisms of drug combinations: interaction and network perspectives. Nat Rev Drug Discov. 2009;8(2):111-28.

8. Park HK, Lee JE, Lim J, Jo DE, Park SA, Suh PG, et al. Combination treatment with doxorubicin and gamitrinib synergistically augments anticancer activity through enhanced activation of Bim. BMC Cancer. 2014;14:431. 
9. Wen C, Fu L, Huang J, Dai Y, Wang B, Xu G, et al. Curcumin reverses doxorubicin resistance via inhibition the efflux function of ABCB4 in doxorubicin-resistant breast cancer cells. Mol Med Rep. 2019;19(6):5162-8.

10. Chen S, Gao J, Halicka HD, Huang X, Traganos F, Darzynkiewicz $\mathrm{Z}$. The cytostatic and cytotoxic effects of oridonin (Rubescenin), a diterpenoid from Rabdosia rubescens, on tumor cells of different lineage. Int J Oncol. 2005;26(3):579-88.

11. Tian L, Xie K, Sheng D, Wan X, Zhu G. Antiangiogenic effects of oridonin. BMC Complement Altern Med. 2017;17(1):192.

12. Tan W, Lu J, Huang M, Li Y, Chen M, Wu G, et al. Anti-cancer natural products isolated from chinese medicinal herbs. Chin Med. 2011;6(1):27.

13. Xia S, Zhang X, Li C, Guan H. Oridonin inhibits breast cancer growth and metastasis through blocking the Notch signaling. Saudi Pharm J. 2017;25(4):638-43.

14. Yao Z, Xie F, Li M, Liang Z, Xu W, Yang J, et al. Oridonin induces autophagy via inhibition of glucose metabolism in p53mutated colorectal cancer cells. Cell Death Dis. 2017;8(2):e2633.

15. Li J, Wu Y, Wang D, Zou L, Fu C, Zhang J, et al. Oridonin synergistically enhances the anti-tumor efficacy of doxorubicin against aggressive breast cancer via pro-apoptotic and anti-angiogenic effects. Pharmacol Res. 2019;146:104313.

16. Wang XH, Zhang SF, Bao JT, Liu FY. Oridonin synergizes with Nutlin-3 in osteosarcoma cells by modulating the levels of multiple Bcl-2 family proteins. Tumour Biol. 2017;39(6):1010428317701638.

17. Lu Y, Sun Y, Zhu J, Yu L, Jiang X, Zhang J, et al. Oridonin exerts anticancer effect on osteosarcoma by activating PPAR- $\gamma$ and inhibiting Nrf2 pathway. Cell Death Dis. 2018;9(1):15.

18. Du Y, Zhang J, Yan S, Tao Z, Wang C, Huang M, et al. Oridonin inhibits the proliferation, migration and invasion of human osteosarcoma cells via suppression of matrix metalloproteinase expression and STAT3 signalling pathway. J BUON. 2019;24(3):1175-80.

19. Fogh J, Trempe G. New human tumor cell lines. In: Fogh J, editor. Human tumor cells in vitro. Boston, MA: Springer; 1975. p. $115-59$.
20. Chou TC. Drug combination studies and their synergy quantification using the Chou-Talalay method. Cancer Res. 2010;70(2):440-6.

21. Sivandzade F, Bhalerao A, Cucullo L. Analysis of the mitochondrial membrane potential using the cationic JC-1 dye as a sensitive fluorescent probe. Bio Protoc. 2019;9(1):e3128.

22. Tsang WP, Chau SP, Kong SK, Fung KP, Kwok TT. Reactive oxygen species mediate doxorubicin induced $\mathrm{p} 53$-independent apoptosis. Life Sci. 2003;73(16):2047-58.

23. Snezhkina AV, Kudryavtseva AV, Kardymon OL, Savvateeva MV, Milnikova NV, Krasnov GS, et al. ROS generation and antioxidant defense systems in normal and malignant cells. Oxid Med Cell Longev. 2019;2019:6175804.

24. Zhu J, Yu W, Liu B, Wang Y, Shao J, Wang J, et al. Escin induces caspase-dependent apoptosis and autophagy through the ROS/p38 MAPK signalling pathway in human osteosarcoma cells in vitro and in vivo. Cell Death Dis. 2017;8(10):e3113.

25. Demelash A, Pfannenstiel LW, Liu L, Gastman BR. Mcl-1 regulates reactive oxygen species via NOX4 during chemotherapyinduced senescence. Oncotarget. 2017;8(17):28154-68.

26. Dong X, Liu F, Li M. Inhibition of nuclear factor $\kappa \mathrm{B}$ transcription activity drives a synergistic effect of cisplatin and oridonin on HepG2 human hepatocellular carcinoma cells. Anticancer Drugs. 2016;27:286-99.

27. He Z, Xiao X, Li S, Guo Y, Huang Q, Shi X, Wang X, Liu $\mathrm{Y}$. Oridonin induces apoptosis and reverses drug resistance in cisplatin resistant human gastric cancer cells. Oncol Lett. 2017;14:2499-504.

Publisher's Note Springer Nature remains neutral with regard to jurisdictional claims in published maps and institutional affiliations. 